\title{
Non-LTE abundances of magnesium, aluminum and sulfur in OB stars near the solar circle
}

\author{
S. Daflon ${ }^{1}$, K. Cunha ${ }^{1}$, V. V. Smith ${ }^{2}$, and K. Butler ${ }^{3}$ \\ 1 Observatório Nacional, Rua General José Cristino 77 CEP 20921-400, Rio de Janeiro, Brazil \\ e-mail: daflon@on.br;katia@on.br \\ 2 Department of Physics, University of Texas at El Paso, El Paso, TX 79968-0515, USA \\ e-mail: verne@barium.physics.utep.edu \\ ${ }^{3}$ Institut für Astronomie und Astrophysik der Universität München, Scheinerstrasse 1, 81679 München, Germany \\ e-mail: butler@usm.uni-muenchen.de
}

Received 15 October 2002 / Accepted 29 November 2002

\begin{abstract}
Non-LTE abundances of magnesium, aluminum and sulfur are derived for a sample of 23 low- $v \sin i$ stars belonging to six northern OB associations of the Galactic disk within $1 \mathrm{kpc}$ of the Sun. The abundances are obtained from the fitting of synthetic line profiles to high resolution spectra. A comparison of our results with HII region abundances indicates good agreement for sulfur while the cepheid abundances are higher. The derived abundances of $\mathrm{Mg}$ show good overlap with the cepheid results. The aluminum abundances for OB stars are significantly below the cepheid values. But, the OB star results show a dependence with effective temperature and need further investigation. The high $\mathrm{Al}$ abundances in the cepheids could be the result of mixing. A discussion of the oxygen abundance in objects near the solar circle suggests that the current mean galactic oxygen abundance in this region is 8.6-8.7 and in agreement with the recently revised oxygen abundance in the solar photosphere. Meaningful comparisons of the absolute $\mathrm{S}, \mathrm{Al}$ and $\mathrm{Mg}$ abundances in $\mathrm{OB}$ stars with the Sun must await a reinvestigation of these elements with 3D hydrodynamical model atmospheres for the Sun. No abundance gradients are found within the limited range in galactocentric distances in the present study. Such variations would be expected only if there were large metallicity gradients in the disk.
\end{abstract}

Key words. stars: abundances - stars: early-type

\section{Introduction}

The massive OB stars, as well as other young objects such as cepheids or H II regions, are used commonly as tracers of the current chemical composition in the Galactic disk. Abundance analyses of $\mathrm{H}$ II regions are restricted to a handful of elements, those being most typically $\mathrm{He}, \mathrm{N}, \mathrm{O}, \mathrm{S}, \mathrm{Ne}$, and Ar. In the OB stars, besides $\mathrm{N}, \mathrm{O}$, and $\mathrm{S}$, the additional elements $\mathrm{C}, \mathrm{Mg}$, $\mathrm{Al}, \mathrm{Si}$, and $\mathrm{Fe}$ can be analyzed using both LTE and non-LTE techniques. Cepheids are the more evolved, cooler descendents of a subset of the OB stars (with masses of $\sim 5-10 M_{\odot}$ ) and a rather large number of elements $(\sim 25)$ can be detected in their spectra. As all three of these types of objects are young $\left(\leq\right.$ few $\left.\times 10^{7} \mathrm{yr}\right)$, it is reasonable to expect that examples of them inhabiting the same region of the Galaxy should contain approximately the same mixture of chemical elements. This approximation should be as good as allowed by small scale chemical inhomogeneities that can be produced on timescales of the lifetimes of large, starforming regions, i.e. a few times $10^{7} \mathrm{yr}$.

Care must be taken in comparing abundances between H II regions, OB stars, and cepheids, however. For example, internal

Send offprint requests to: S. Daflon stellar mixing may affect both OB stars and cepheids to varying degrees. Some evidence of the mixing of material exposed to the $\mathrm{CN}$-cycle has been uncovered in certain OB stars, even near the main sequence (Gies \& Lambert 1992); this mixing may be driven primarily by rotation (Heger \& Langer 2000). In this case, nitrogen abundances are measurably enhanced (by $\sim+0.3$ dex) with, perhaps, a marginal decrease in the carbon abundance (by $\sim-0.1$ dex), while oxygen remains untouched. In the more evolved cepheids, even deeper mixing may have occurred that involves the full CNO cycles, such that some oxygen depletion might be detectable (as well as larger $\mathrm{N}$ enhancements and $\mathrm{C}$ depletions). There is also the possibility that surface abundances of sodium, magnesium, and aluminum have been altered by the mixing of material exposed to the $\mathrm{Ne}-\mathrm{Na}$ and $\mathrm{Mg}-\mathrm{Al}$ cycles. In the $\mathrm{H}$ II regions, uncertainties or systematics may arise from temperature fluctuations in the gas, unknown radiation environments, or possible depletions of some elements out of the gas phase and onto solid particles.

By concentrating on a set of $\mathrm{OB}$ stars, in comparison to sample H II regions and cepheids, all near the solar circle, abundance trends found in these various objects can be intercompared and ultimately be checked against their 
corresponding solar values. Such checks can help uncover possible inconsistencies and provide stronger constraints on abundance gradients derived from different types of objects. That is the aim of this paper, which is the fourth paper in a series whose ultimate goal is to use a large sample of OB stars to trace abundance gradients in the Galactic disk. Paper I (Daflon et al. 1999) presented the first results from this survey and concentrated on 8 sharp lined $\operatorname{star}\left(v \sin i \leq 60 \mathrm{~km} \mathrm{~s}^{-1}\right)$ members of the Cep OB2 association: LTE abundances of C, N, O, Si, and $\mathrm{Fe}$ were derived, as well as non-LTE abundances of $\mathrm{C}$, N, O, and Si. Daflon et al. (2001a - Paper II) added analyses of 15 members of five additional northern OB associations (Cep OB3, Cyg OB2, Cyg OB7, Lac OB1, and Vul OB1). The same sets of elements were analyzed in non-LTE as in Paper I, but additional LTE results were presented for $\mathrm{Mg}$, Al, and S. In Paper III (Daflon et al. 2001b), the analysis was expanded to include some of the more rapidly rotating stars $\left(v \sin i=60-150 \mathrm{~km} \mathrm{~s}^{-1}\right)$ in the northern sample. In addition, the atomic analysis included non-LTE calculations for $\mathrm{Mg}$ and $\mathrm{Al}$. In this paper, the non-LTE calculations for $\mathrm{Mg}$ and $\mathrm{Al}$ are applied to the remaining northern stars, and sulfur is now added as an element that can be studied in non-LTE.

\section{Observations and stellar parameters}

The observational data consist of high resolution $(R \sim 60000)$, high signal-to-noise spectra of 23 main sequence late-O/early$\mathrm{B}$ stars belonging to the $\mathrm{OB}$ associations of Cep OB2, Cep OB3, Cyg OB3, Cyg OB7, Vul OB1 and Lac OB1. The spectra were obtained at the McDonald Observatory, University of Texas, Austin, with the $2.1 \mathrm{~m}$ telescope plus the Sandiford Echelle Spectrograph. A set of lower resolution $(R=$ $12000)$ spectra of these targets were obtained with the $2.7 \mathrm{~m}$ telescope plus a Coudé Spectrograph in the $\mathrm{H} \gamma$ region. More details about the observations and data reduction are found in Papers I and II. Also in these studies are derived the stellar parameters and microturbulences for the 23 target stars.

The effective temperatures and surface gravities have been derived from a photometric calibration for the reddening-free index $\mathrm{Q}$ coupled to the fitting of the broadened wings of $\mathrm{H} \gamma$ profile. In our earlier series of papers (Papers I and II) it has been argued that this method results in uncertainties of $\sim 4 \%$ in $T_{\text {eff }}$ and $\pm 0.10 \mathrm{dex}$ in $\log g$. The uncertainties in gravity can be also viewed by noting the positions of stars in a $\log T_{\text {eff }}-\log g$ diagram in comparison to model tracks. For stars of these effective temperatures the $\log g$ of the ZAMS is about 4.2. An average of all surface gravities of stars here that are not clearly evolved (i.e., $\log g \leq 4.0$ ) finds $\log g=4.25 \pm 0.17$; close to what is expected for stars near the main sequence with a scatter larger than what we have estimated as the expected uncertainty. The microturbulent velocities were obtained from the requirement that the non-LTE O II abundances were independent of the line-strength. The sample stars, their corresponding associations and spectral types, adopted effective temperatures, surface gravities and $\xi$-value are gathered in Table 1 . We note that the surface gravity for the star HD 214167 has been revised.
Table 1. Stellar parameters.

\begin{tabular}{cccccc}
\hline \hline Association & Star & MK & $T_{\text {eff }}(\mathrm{K})$ & $\log g$ & $\xi\left(\mathrm{km} \mathrm{s}^{-1}\right)$ \\
\hline CepOB2 & HD 205794 & B0.5V & 26890 & 4.21 & 8.0 \\
& HD 206183 & O9.5V & 33310 & 4.52 & 5.0 \\
& HD 206267 & B0V & 26100 & 4.21 & 5.0 \\
& HD 206327 & B2V & 21900 & 3.99 & 8.0 \\
& HD 207538 & O9V & 32190 & 4.32 & 10.0 \\
& HD 239724 & B1V & 24790 & 3.83 & 12.0 \\
& HD 239742 & B2V & 22470 & 4.07 & 5.0 \\
& HD 239743 & B2V & 21580 & 3.99 & 8.0 \\
VulOB1 & BD + 24 3880 & B0.5V & 30410 & 4.57 & 8.0 \\
& HD 344783 & B0IV & 31010 & 4.26 & 9.0 \\
CygOB3 & HD 227460 & B1IV & 27060 & 4.34 & 8.0 \\
& HD 227586 & B0.5V & 27830 & 4.15 & 8.0 \\
& HD 227757 & O9.5V & 32480 & 4.22 & 8.0 \\
CygOB7 & HD 197512 & B1V & 23570 & 4.02 & 6.0 \\
& HD 202253 & B1.5IV & 22750 & 3.95 & 6.0 \\
LacOB1 & HD 214167 & B1V & 26720 & 4.54 & 6.0 \\
& HD 214680 & O9V & 33690 & 4.27 & 11.0 \\
& HD 216916 & B2IV & 23520 & 4.00 & 6.0 \\
& HD 217227 & B2V & 19000 & 4.20 & 7.0 \\
& HD 217811 & B2V & 19070 & 3.92 & 5.0 \\
CepOB3 & BD + 62 2125 & B1.5V & 23480 & 4.05 & 10.0 \\
& HD 217657 & B0.5V & 27950 & 4.38 & 8.0 \\
& HD 218342 & B0IV & 30020 & 4.20 & 9.0 \\
\hline
\end{tabular}

Table 2. Linelists.

\begin{tabular}{|c|c|c|c|c|}
\hline Wavelength interval & $\lambda(\AA)$ & Species & $\chi(\mathrm{eV})$ & $\log (g f)$ \\
\hline $4359-4367$ & 4361.53 & S III & 18.24 & -0.75 \\
\hline \multirow{2}{*}{ S III } & 4364.75 & S III & 18.32 & -0.85 \\
\hline & 4366.91 & O II & 23.01 & -0.24 \\
\hline $4476-4484$ & 4479.88 & Al III & 20.78 & $0.90 *$ \\
\hline \multirow[t]{4}{*}{ Mg II, Al III } & 4479.97 & Al III & 20.78 & $1.02 *$ \\
\hline & 4481.13 & $\mathrm{Mg}$ II & 8.86 & 0.74 \\
\hline & 4481.15 & $\mathrm{Mg}$ II & 8.86 & -0.56 \\
\hline & 4481.33 & $\mathrm{Mg}$ II & 8.86 & 0.59 \\
\hline $4510-4515$ & 4510.88 & N III & 35.67 & -0.45 \\
\hline \multirow[t]{2}{*}{$\mathrm{Al}$ III } & 4512.56 & Al III & 17.81 & 0.40 \\
\hline & 4514.85 & N III & 35.70 & 0.23 \\
\hline $4526-4531$ & 4527.86 & N III & 38.49 & -0.24 \\
\hline \multirow[t]{2}{*}{ Al III } & 4529.19 & Al III & 17.81 & 0.66 \\
\hline & 4530.41 & N II & 23.49 & 0.67 \\
\hline
\end{tabular}

${ }^{*} \log (g f)$ from Kurucz web site; all the others $g f$-values are from TOPbase.

\section{Non-LTE abundances}

Non-LTE synthetic spectra were calculated for the few transitions of Mg II, Al III and S III that are available in the spectra of early-type stars. Although relatively free of blends, linelists for each spectral region containing the lines of interest were constructed within the interval around $\pm 3 \AA$. These transitions are listed in Table 2 together with their wavelengths, designations, excitation potentials as well as adopted $g f$-values. 
The abundance analyses in this study are based on the fullyblanketed and plane-parallel LTE model atmospheres calculated with the ATLAS9 code (Kurucz 1992) for a constant microturbulent velocity of $\xi=2 \mathrm{~km} \mathrm{~s}^{-1}$ and solar composition. Departures from LTE were considered in the line formation calculations with the newest version of the program DETAIL (Butler 1994). The adopted model atoms are described in Przybilla et al. (2001-Mg II), Dufton et al. (1986Al III) and Vrancken et al. (1996-S III). We note that so far, all abundance papers including non-LTE line formation of Al published in the literature (Vrancken et al. 1997; Vrancken et al. 2000; Gummersbach et al. 1998) were based on the same model atom adopted in this study, and in a sense are not completely independent. Published non-LTE abundance analyses of magnesium (Vrancken et al. 1997; Vrancken et al. 2000; Gummersbach et al. 1998) are based on the model atom of Mihalas (1972). All sulfur abundances in the literature are derived from LTE analysis, except for Vrancken et al. (1996), who analyzed three B stars as a test for their sulfur model atom. The present study is the first systematic non-LTE analysis of sulfur abundances in OB stars.

A brief description of the adopted model atoms follows. Przybilla et al. (2001) constructed an extensive model atom for $\mathrm{Mg} \mathrm{I} / \mathrm{Mg}$ II based on recent atomic data. This model is roughly complete for levels up to $n=9$ for $\mathrm{Mg}$ I and $n=10$ for $\mathrm{Mg}$ II, whereas $\mathrm{Mg}$ III, that does not have a significant population in the range of effective temperatures considered here, is represented only by its ground state. The sulfur model atom (Vrancken et al. 1996) treats S II/S III simultaneously considering 81 levels of S II and 21 levels of S III. The model atom also includes the three lowest levels of S I and the two lowest levels of S IV, together with the ground state of S V that are important for the hotter stars. Vrancken et al. (1996) tested their model atom for three B2 III-V stars and concluded that S III lines yield more reliable abundances (than S II lines) for the temperature range they consider. The adopted Al III model atom of Dufton et al. (1986) is less complete than the magnesium and sulfur ones. It consists of 12 states of Al III plus the ground state of $\mathrm{Al}$ IV, as the populations of $\mathrm{Al}$ II and $\mathrm{Al} \mathrm{V}$ are not significant in the temperature range between 20000 to $35000 \mathrm{~K}$.

The synthetic line profiles were calculated with the SURFACE code (Butler 1984), assuming Voigt profile functions. These profiles were then broadened by means of convolution with the rotational profile, including $v \sin i$ and $\operatorname{limb}$ darkening, and instrumental profile. The microturbulence for each target star was adopted from O II lines, derived in previous studies, while the line abundances and $v \sin i$ 's were allowed to vary. The best fit was chosen from the $\chi^{2}$-minimization of the differences between theoretical and observed profiles. The final non-LTE abundances and $v$ sin $i$ 's are listed in Table 3 . The abundances are represented by the average of the individual line abundances and the respective dispersions (and number $[n]$ of fitted lines), whenever this is the case. A sample of profile fitting for all the spectral regions is shown in the panels of Fig. 1, for the star HD 197512.

In general, the published non-LTE $\mathrm{Mg}, \mathrm{Al}$ and $\mathrm{S}$ abundances are consistent with our results. However, aluminum abundances deserve special attention. As a test, we recalculated
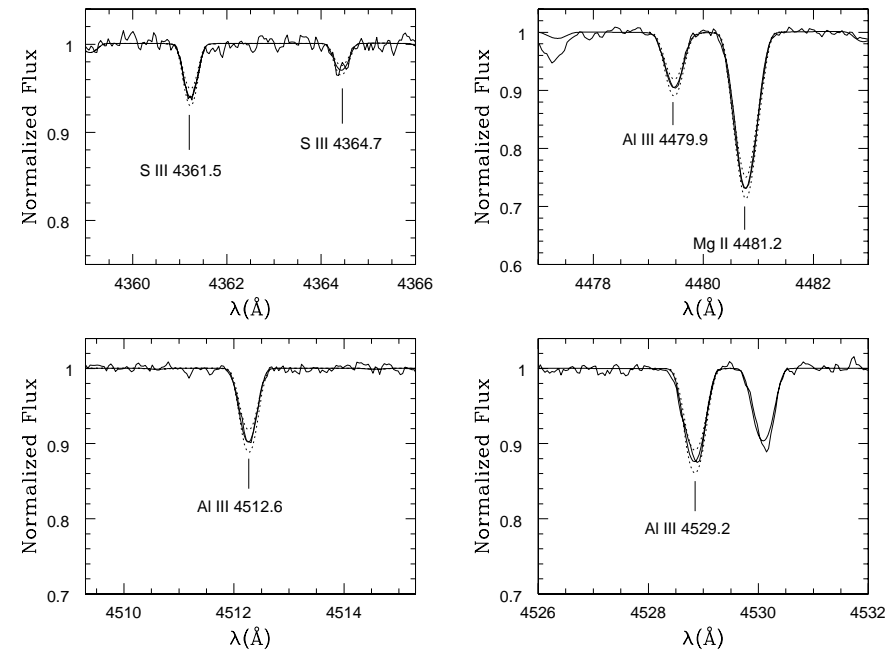

Fig. 1. Some examples of line synthetic profiles fitted to the observed spectra of the star HD 197512 for $\xi=6 \mathrm{~km} \mathrm{~s}^{-1}$. The solid lines represent the best fit obtained for each observed line profile, identified by its wavelength in $\AA$, whereas the dotted lines depict the theoretical profiles computed for abundances sligthly lower and higher $( \pm 0.1 \mathrm{dex})$ than the adopted value for each element.

the non-LTE abundances of star 201 in NGC 2244, analyzed by Vrancken et al. (1997), using their listed equivalent widths and, as expected, we reproduced their derived abundance within the errors. We also compared our non-LTE Al abundances with those interpolated in the grid of theoretical equivalent widths of Dufton et al. (1986). The comparison for the model atmospheres with lower $T_{\text {eff }}$ showed that our abundances derived directly from synthesis agree within the uncertainties with the abundances interpolated within their grid. For the hottest models, however, Dufton's grid yields aluminum abundances much lower than 6.0. The difference between our synthetic profiles and Dufton's grid resides basically in the model atmospheres, as they used non-LTE non-blanketed model atmospheres and we use fully blanketed LTE models from Kurucz (1992).

Errors in the determination of the stellar parameters, microturbulence, $v \sin i$ and the placement of the continuum are the main sources of uncertainties in a chemical analysis based on the fitting of line profiles. In Paper III we discussed the uncertainties assigned for each of these parameters and the subsequent abundance error arising from them. Accordingly, our total errors expected for the derived magnesium abundances are of the order of 0.2 dex, being larger for the coolest stars $(0.3 \mathrm{dex}$ for $T_{\text {eff }} \sim 19000 \mathrm{~K}$ ) and these are dominated by the uncertainty in the microturbulent velocity (as the abundance analysis is based on one intermediate to strong line) and effective temperature. The uncertainties in sulfur abundances arise chiefly from the errors in $T_{\text {eff }}$ and are estimated to be of the order of $\sim 0.15$ dex. The profile of the weak S III line at $4364 \AA$ was only fitted for the stars with lowest $v \sin i$ and highest signal-to-noise spectra. For this reason, the sulfur abundances of some stars in our sample are based only on the S III line at $4361 \AA$. Formally, aluminum presents smaller errors, around 0.10 dex. However, the abundances derived from the lines $\lambda \lambda 4512$ and $4529 \AA$, that belong to the same multiplet, show some dependence with 
Table 3. Non-LTE Mg, Al, and S abundances.

\begin{tabular}{cccccc}
\hline \hline Association & Star & $v \sin i\left(\mathrm{~km} \mathrm{~s}^{-1}\right)$ & $\log \epsilon(\mathrm{Mg})[n]$ & $\log \epsilon(\mathrm{Al})[n]$ & $\log \epsilon(S)[n]$ \\
\hline CepOB2 & HD 205794 & $13.2 \pm 1.2$ & $7.33[1]$ & $5.81 \pm 0.16[2]$ & $7.34[1]$ \\
& HD 206183 & $19.2 \pm 1.9$ & $7.36[1]$ & $6.06 \pm 0.19[3]$ & $7.05[1]$ \\
& HD 206267 & $47.0 \pm 3.0$ & $7.21[1]$ & $5.74 \pm 0.15[3]$ & $7.28[1]$ \\
& HD 206327 & $20.6 \pm 2.1$ & $7.27[1]$ & $5.98 \pm 0.05[3]$ & $7.17 \pm 0.05[2]$ \\
& HD 207538 & $42.8 \pm 2.0$ & $7.42[1]$ & $6.15 \pm 0.39[2]$ & $\ldots$ \\
& HD 239724 & $38.0 \pm 1.0$ & $7.48[1]$ & $5.79 \pm 0.24[3]$ & $7.16 \pm 0.16[2]$ \\
& HD 239742 & $9.5 \pm 1.2$ & $7.37[1]$ & $5.96 \pm 0.07[3]$ & $7.19[1]$ \\
& HD 239743 & $23.2 \pm 1.3$ & $7.33[1]$ & $6.01 \pm 0.03[3]$ & $\ldots$ \\
VulOB1 & BD $24{ }^{\circ} 3880$ & $12.9 \pm 3.3$ & $7.59[1]$ & $6.24 \pm 0.19[3]$ & $7.28 \pm 0.08[2]$ \\
& HD 344783 & $29.2 \pm 4.3$ & $7.31[1]$ & $5.95 \pm 0.42[2]$ & $7.13 \pm 0.03[2]$ \\
CygOB3 & HD 227460 & $14.2 \pm 2.8$ & $7.46[1]$ & $6.11 \pm 0.17[3]$ & $7.22[1]$ \\
& HD 227586 & $19.7 \pm 3.2$ & $7.21[1]$ & $5.79 \pm 0.11[2]$ & $7.21[1]$ \\
& HD 227757 & $29.6 \pm 3.2$ & $7.39[1]$ & $6.29 \pm 0.23[3]$ & $7.34[1]$ \\
CygOB7 & HD 197512 & $16.5 \pm 1.2$ & $7.38[1]$ & $5.98 \pm 0.07[3]$ & $7.11 \pm 0.19[2]$ \\
& HD 202253 & 48 & $\ldots$ & $5.96[1]$ & $\ldots$ \\
LacOB1 & HD 214167 & $21.6 \pm 4.4$ & $7.57[1]$ & $6.01 \pm 0.09[3]$ & $7.09[1]$ \\
& HD 214680 & $27.2 \pm 4.5$ & $7.47[1]$ & $6.28 \pm 0.31[3]$ & $\ldots$ \\
& HD 216916 & $14.3 \pm 3.3$ & $7.52[1]$ & $6.12 \pm 0.16[3]$ & $7.29[1]$ \\
& HD 217227 & $18.5 \pm 2.4$ & $7.52[1]$ & $6.18 \pm 0.08[3]$ & $7.28[1]$ \\
& HD 217811 & $9.4 \pm 0.6$ & $7.46[1]$ & $6.05 \pm 0.04[3]$ & $7.11 \pm 0.18[2]$ \\
CepOB3 & BD + 62 2125 & $39.6 \pm 3.2$ & $7.03[1]$ & $5.84 \pm 0.01[2]$ & $7.20 \pm 0.21[2]$ \\
& HD 217657 & $17.4 \pm 2.4$ & $7.12[1]$ & $5.71 \pm 0.11[2]$ & $7.17[1]$ \\
& HD 218342 & $35.2 \pm 4.8$ & $7.44[1]$ & $5.99 \pm 0.01[2]$ & $7.06 \pm 0.08[2]$ \\
\hline
\end{tabular}

effective temperature and introduce a larger discrepancy in the average abundances of the hottest stars.

\section{Discussion}

OB stars, as well as other young objects, such as H II regions, track the chemical composition of the Galactic disk. With this paper, we now have a uniform set of non-LTE abundances for $\mathrm{C}, \mathrm{N}, \mathrm{O}, \mathrm{Mg}, \mathrm{Al}, \mathrm{Si}$, and $\mathrm{S}$ in 35 stars of the northern sample. All six OB associations that are represented in this sample lie within $1 \mathrm{kpc}$ of the solar circle (where we take $R_{\odot}=7.9 \mathrm{kpc}$, McNamara et al. 2000) and, as such, will have abundances that are, in principle, not affected significantly by possibly modest galactic abundance gradients. The abundances of certain elements like sulfur, magnesium and aluminum, as well as oxygen can be compared to recent results derived from cepheids and $\mathrm{H}$ II regions also lying within the same galactocentric distance interval.

The stars in this sample are all sharp-lined stars, with $v \sin i \leq 48 \mathrm{~km} \mathrm{~s}^{-1}$. The mean magnesium abundance is $A(\mathrm{Mg})=7.37 \pm 0.14$. The magnesium abundances are quite independent of effective temperature, as shown in the top panel of Fig. 2. Aluminum shows significantly lower abundances in the OB stars than the Sun, as displayed in the middle panel of Fig. 2. Such a large difference may reside partially in uncertainties in the non-LTE calculations for Al III. Some evidence for this possibility may appear in the derived aluminum abundances showing a small offset between the hotter and cooler stars. There is a noticeable displacement between stars with $T_{\text {eff }} \geq 30000 \mathrm{~K}$ and those with lower effective temperatures, with the hotter sample having a mean aluminum abundance of
$6.13 \pm 0.14$ and the cooler having $5.94 \pm 0.14$. For the S III nonLTE abundances, there is no trend with $T_{\text {eff }}$ and the scatter is small: the mean $A(S)=7.19 \pm 0.09$ is 0.14 dex below the solar abundance of $7.33 \pm 0.11$ (Grevesse \& Sauval 1998).

Taken together, these new results for $\mathrm{S}, \mathrm{Mg}$ and $\mathrm{Al}$, could in principle suggest that the OB stars, on average, are slightly underabundant when compared to the Sun. However, these differences could be argued to be at the level of systematics. In fact, the recent results from 3D-hydrodynamical model atmosphere calculations for the elements $\mathrm{C}, \mathrm{N}$ and $\mathrm{O}$ in the solar photosphere have lowered their abundances significantly. The photospheric carbon, nitrogen and oxygen abundances, for instance, have been recently revised to $8.41,7.80$, and 8.66 , respectively (Asplund 2002), and are now in better agreement with the results for OB stars. In addition, the OB results rely on 1D-models while the recent solar results are from 3D hydrodynamical models. Although it is expected that the radiative OB atmospheres will not have temperature inhomogeneities that are present in the solar atmosphere (caused by convection) it is possible that some other effects like the high microturbulent velocities required for the $\mathrm{OB}$ stars indicates that $1 \mathrm{D}$ models are not a complete description of atmospheres of hot stars.

Abundances for the different types of young objects, OB stars, cepheids, and H II regions, are compared in Fig. 3 for the elements $\mathrm{Mg}, \mathrm{Al}$, and $\mathrm{S}$, as well as $\mathrm{O}$ (with oxygen taken from our previous papers). We restrict our discussion to galactocentric distances that overlap those values found for the six OB associations represented here, where $R_{\mathrm{g}}=6.9$ to $8.2 \mathrm{kpc}$. Oxygen is added to the discussion in Fig. 3 as it represents the most abundant element after $\mathrm{H}$ and $\mathrm{He}$, and is the element 


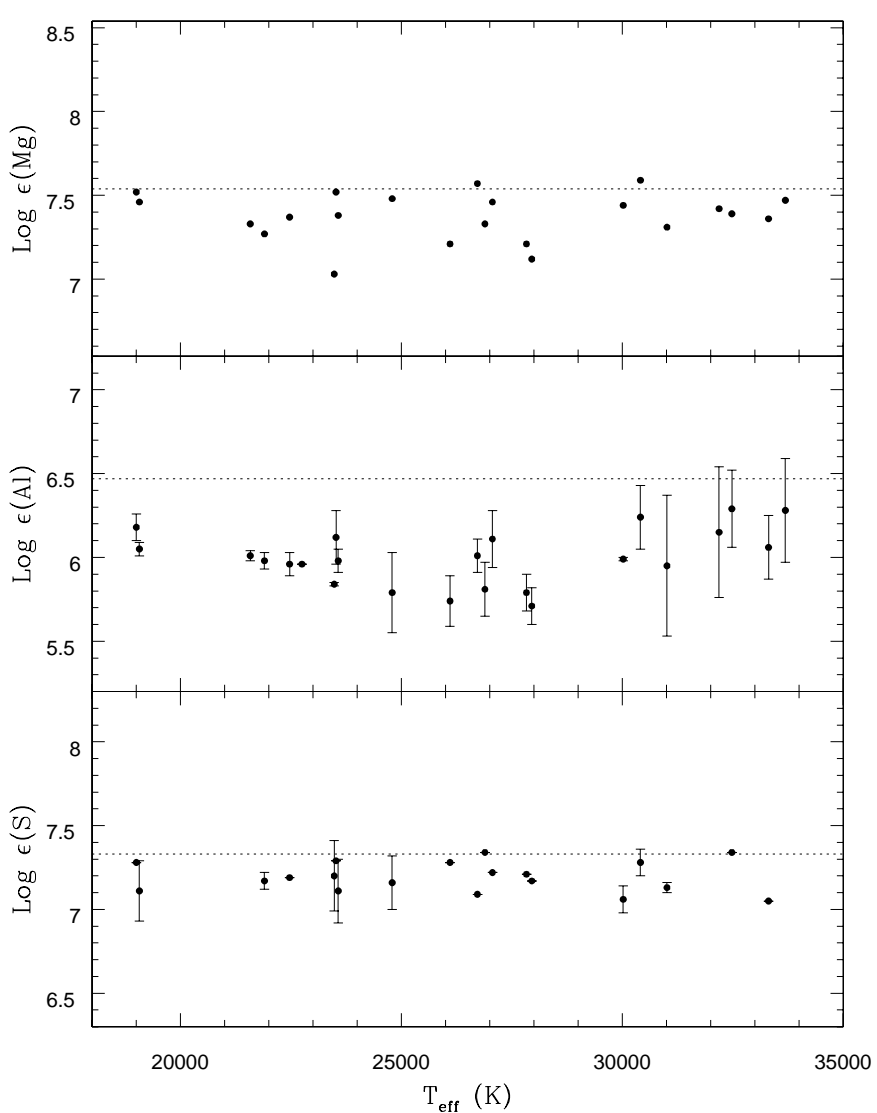

Fig. 2. Elemental abundances of magnesium, aluminum and sulfur as a function of effective temperature. The abundances are represented by the average of individual lines while errorbars represent the respective standard deviations. The dotted lines represent the solar abundances listed by Holweger (2001, Mg: $7.538 \pm 0.060)$ and Grevesse \& Sauval (1998, Al: $6.47 \pm 0.07$, S: $7.33 \pm 0.11)$.

comprising the largest fraction of a star's abundance of heavy elements. In Fig. 3, the mean abundances of each OB association are plotted as filled squares, with the errorbars representing the standard deviation found within the abundances of the association members (in the case where only 2 members were represented, the errorbars are the average differences from the mean). Over the limited range of distance sampled by this particular set of stars, no significant abundance gradient is apparent and the horizontal solid lines are the mean abundances of the set of $\mathrm{OB}$ associations. The solar symbol is plotted at $R_{\odot}=7.9 \mathrm{kpc}$. The mean oxygen abundance (non-LTE calculations for O II from Papers I, II, and III) is $A(\mathrm{O})=8.58 \pm 0.09$. Again, as discussed above, in agreement with the revised solar value.

Other results plotted in Fig. 3 include the recent cepheid results from Andrievsky et al. (2002), shown as open circles, and H II regions from the optical emission lines analyzed by Deharveng et al. (2000), shown as six-pointed stars, and H II region far-IR fine-structure lines by Simpson et al. (1995) and Afflerbach et al. (1997), shown as three-pointed stars.

Beginning with oxygen in Fig. 3, with abundances shown for cepheids and $\mathrm{H}$ II region optical and far-IR lines, there is considerable overlap among all sets of results. The cepheid O-abundances tend to be slightly higher than the
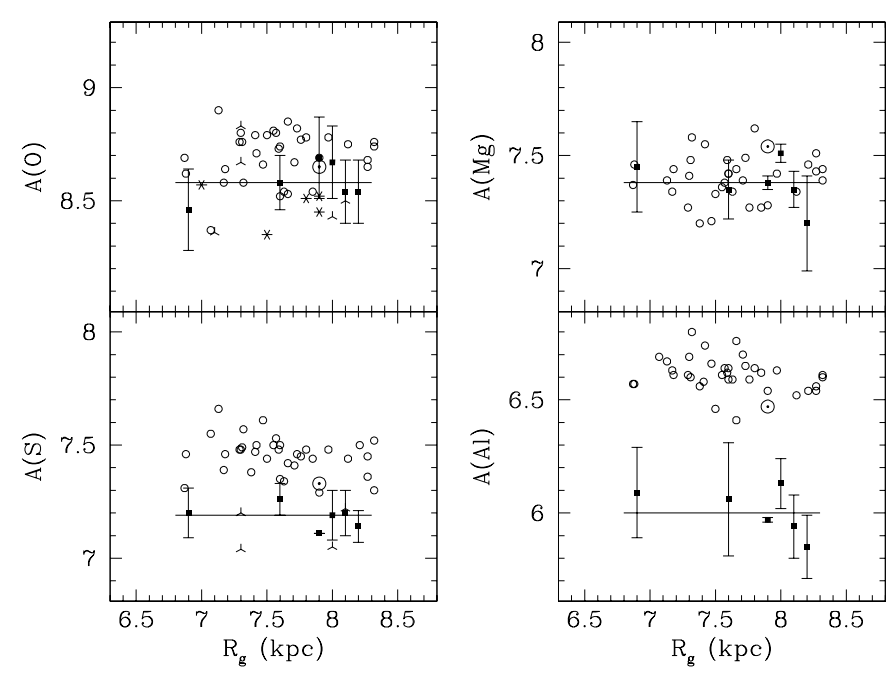

Fig. 3. A comparison between abundances for OB stars, cepheids, and $\mathrm{H}$ II regions, for the elements $\mathrm{O}, \mathrm{Mg}, \mathrm{Al}$, and $\mathrm{S}$. The abundances are plotted as a function of the Galactocentric distance, within $1 \mathrm{kpc}$ from the Sun, represented at $R_{\odot}=7.9 \mathrm{kpc}$. The mean abundances of each $\mathrm{OB}$ association are plotted as filled squares, with the errorbars representing the standard deviation. The straight lines depict the average abundance for the six OB associations for each element. Abundances for cepheids (Andrievsky et al. 2002) are shown as open circles. The abundances for H II regions are represented by six-pointed stars (Deharveng et al. 2000), and three-pointed stars (Simpson et al. 1995; Afflerbach et al. 1997).

OB associations, but only by $\sim 0.1$ dex: likely within possible systematic offsets. The optical H II region results from Deharveng et al. (2000) scatter almost perfectly within the OB association results, while the far-IR results exhibit a larger scatter, but with an average $\mathrm{O}$-abundance that agrees with the $\mathrm{OB}$ associations. Overall, the suggestion from all of the oxygen abundances is that the current mean Galactic abundance near the solar circle is about $A(\mathrm{O}) \sim 8.6-8.7$, with an intrinsic dispersion that is not yet well-defined. The dispersion among the six OB associations is quite small (as well as the $\mathrm{H}$ II region optical emission-line results).

The panel of $\mathrm{Mg}$ and $\mathrm{Al}$ abundances in Fig. 3 contains only $\mathrm{OB}$ association and cepheid results. In the case of $\mathrm{Mg}$, the overlap between OB stars and cepheids is essentially perfect. The mean and standard deviations are $A(\mathrm{Mg})=7.37 \pm 0.14$ for the $\mathrm{OB}$ associations and $7.36 \pm 0.10$ for the cepheids. This tidy agreement between cepheids and $\mathrm{OB}$ stars for $\mathrm{Mg}$ is not repeated for $\mathrm{Al}$, where the cepheid $\mathrm{Al}$ abundances are measurably above solar, while the OB stars are well below solar. As discussed previously, the OB star abundances are based upon non-LTE calculations using Al III lines and there is a noticeable offset in the abundances derived for the hotter and cooler stars. This may indicate problems in the non-LTE calculations. On the other hand, the rather large $\mathrm{Al}$ abundances for the cepheids may not be intrinsic, but could be the result of stellar mixing involving material exposed to the $\mathrm{Mg}$ - $\mathrm{Al}$ cycle of $\mathrm{H}$-burning. An increase in aluminum would come from a decrease in $\mathrm{Mg}$, however, the observed offset in the $\mathrm{Al}$ abundances seen in the cepheids would result in a negligible decrease in $\mathrm{Mg}$, due to the 
larger $\mathrm{Mg}$ abundance. For example, if the current Galactic Al abundance is about solar, then the cepheids have had their Al abundances enhanced by $\sim 0.2$ dex. If this enhancement came from $\mathrm{Mg}$ atoms, it would result in a decrease in the overall $\mathrm{Mg}$ abundance by $\sim 0.02-0.03$ dex, which is effectively unmeasurable. More work will be needed to decide the best value for the current Galactic aluminum abundance at the solar circle.

Finally, Fig. 3 also shows sulfur abundances derived from the OB stars, cepheids, and H II regions (from the far-IR lines). Here, the OB associations and $\mathrm{H}$ II regions again show a great degree of overlap. The cepheids exhibit systematically larger $\mathrm{S}$ abundances than the Sun, and these enhancements cannot be due to internal stellar mixing.

\section{Conclusions}

We have presented non-LTE abundances of magnesium, aluminum and sulfur for $23 \mathrm{OB}$ stars members of OB associations within $1 \mathrm{Kpc}$ of the Sun. Magnesium abundances derived for cepheids in the literature agree well with the results for $\mathrm{OB}$ stars while no $\mathrm{Mg}$ abundances can be derived for $\mathrm{H}$ II regions. On the other hand, sulfur results point to discrepencies between the cepheids on one side and the OB stars and H II regions on the other, that are not easily resolved: the cepheid sulfur abundances are derived from S I lines, while the OB stars use S III lines, and the H II region results come from far-IR [S III] lines at $19 \mu \mathrm{m}$. Aluminum abundances OB stars must be interpreted with caution: the relatively low $\mathrm{Al}$ abundances derived may reflect problems in the non-LTE calculations for this species and deserves additional analysis before further conclusions while $\mathrm{Al}$ in the cepheids could be enhanced due to internal mixing.

Acknowledgements. S.D. acknowledges a CAPES fellowship and partial financial support from DAAD (Germany). KC thanks David Lambert for travel support for observing runs in 1992, 1993, and 1994. VVS acknowledges support by the National Science Foundation through grant AST99-87374.

\section{References}

Afflerbach, A., Churchwell, E., \& Werner, M. W. 1997, ApJ, 478, 190 Andrievsky, S. M., Kovtyukh, V. V., Luck, R. E., et al. 2002, A\&A, 381,32

Asplund, M. 2002, to appear in ASP Conf. Ser., CNO in the Universe, ed. C. Charbonnel, D. Schaerer, \& G. Meynet

Butler, K. 1984, Ph.D. Thesis, University of London

Butler, K. 1994, http://ccp7.dur .ac.uk/Docs/detail.ps

Daflon, S., Cunha, K., \& Becker, S. 1999, ApJ, 522, 950 (Paper I)

Daflon, S., Cunha, K., Becker, S., \& Smith, V. V. 2001a, ApJ, 552, 309 (Paper II)

Daflon, S., Cunha, K., Butler, K., \& Smith, V. V. 2001b, ApJ, 563, 325 (Paper III)

Deharveng, L., Peña, M., Caplan, J., \& Costero, R. 2000, MNRAS, 311,329

Dufton, P. L., Brown, P. J. F., Lennon, D. J., \& Lynas-Gray, A. E. 1986, MNRAS, 222, 713

Gies, D. R., \& Lambert, D. L. 1992, ApJ, 387, 673

Grevesse, N., \& Sauval, A. J. 1998, Space Sci. Rev., 85, 161

Gummersbach, C. A., Kaufer, A., Schäfer, D. R., Szeifert, T., \& Wolf, B. 1998, A\&A, 338, 881

Heger, A., \& Langer, N. 2000, ApJ, 544, 1016

Holweger, H. 2001, in Solar and Galactic Composition, ed. R. F. Wimmer-Schweingruber (American Institute of physics), 23

Kurucz, R. L. 1992, ATLAS9 Stellar Atmosphere Programs and $2 \mathrm{Km} / \mathrm{s}$ Grid CD-Rom 13, Smithsonian Astrophysical Observatory, Cambridge

McNamara, D. H., Madsen, J. B., Barnes, J., \& Ericksen, B. F. 2000, PASP, 112, 202

Mihalas, D. 1972, ApJ, 177, 115

Przybilla, N., Butler, K., Becker, S. R., \& Kudritzki, R. P. 2001, A\&A, 369, 1009

Simpson, J. P., Colgan, S. W. J., Rubin, R. H., Erickson, E. F., \& Haas, M. R. 1995, ApJ, 444, 721

Vrancken, M., Butler, K., \& Becker, S. R. 1996, A\&AS, 311, 661

Vrancken, M., Hensberge, H., David, M., \& Verschueren, W. 1997, A\&A, 320, 878

Vrancken, M., Lennon, D. J., Dufton, P. L., \& Lambert, D. L. 2000, A\&A, 358, 639 